\title{
Depression, auditory-verbal hallucinations, and delusions in patients with schizophrenia: different patterns of association with prefrontal gray and white matter volume
}

\begin{abstract}
Structural brain abnormalities, including decreased gray matter (GM) and white matter (WM) volume, have been observed in patients with schizophrenia. These decrements were found to be associated with positive and negative symptoms, but affective symptoms (depression and anxiety) were poorly explored. We hypothesized that abnormalities in GM and WM volume might also be related to affective symptoms. GM and WM volumes were calculated from high-resolution T1 structural images acquired from 24 patients with schizophrenia and 26 healthy controls, and the associations of positive, negative, and affective symptoms with the brain volumes that showed significant reduction in patients were investigated. Patients demonstrated GM volume reductions in the bilateral prefrontal cortex, and WM volume reductions in the right frontal and left corpus callosum. Prefrontal cortex volume was significantly and inversely associated with both auditory-verbal hallucinations and depression severity. WM volume alterations, in contrast, were related to alogia, anhedonia, and delusions. The combined impact of auditory-verbal hallucinations and depression on similar subregions of the prefrontal cortex suggests that depression is involved in hearing voices. Further, this adverse impact of depression on prefrontal GM volume may underlie the impairment demonstrated by these patients in cognitive tasks that rely on executive processes.
\end{abstract}


Key words: prefrontal cortex, depressive symptoms, auditory-verbal hallucinations, delusions.

\section{Introduction}

Schizophrenia is a severe mental illness characterized by brain structural abnormalities. Previous meta-analyses and studies using voxel-based morphometry (VBM) have revealed reduction in Gray Matter (GM) and White Matter (WM) volume in this population. In particular, large GM volume reduction was observed in the prefrontal cortex (Fornito et al., 2009; Haijma et al., 2013; Ohtani et al., 2014b) and temporal cortex (Fornito et al., 2009). These volume reductions have been related to various positive and negative symptoms of schizophrenia.

\subsection{Auditory Verbal Hallucinations}

Auditory Verbal Hallucinations (AVHs) have mostly been related to GM volume reduction in the superior temporal gyrus, involved in the processing of language (Modinos et al., 2013; O’Daly et al., 2007; Palaniyappan et al., 2012; Rajarethinam et al., 2000; Sun et al., 2009). Associations were also observed with reductions in the medial frontal lobe (Kubera et al., 2014), the inferior frontal gyrus (García-Martí et al., 2008; Gaser et al., 2004; Modinos et al., 2013) and other brain areas including the inferior supramarginal gyrus (parietal lobe) (Gaser et al., 2004), the insula (Kubera et al., 2014) and the putamen (Van Tol et al., 2014). AVH have also been related to WM volume decrement, more particularly in the right internal capsule and inferior longitudinal fasciculus - the fiber tract that connects the temporal and occipital lobes (O'Daly et al., 2007), the postcentral gyrus (Van Tol et al., 2014), the occipital lobe, the corpus callosum (Makris et al., 2010), and the superior longitudinal fasciculus, which connects the frontal, occipital and temporal lobes (Seok et al., 2007). 


\subsection{Delusions}

Delusions in patients with schizophrenia have been found to be associated with GM volume reduction in the temporal (Crespo-Facorro et al., 2004; Song et al., 2015). and frontal (Maruff et al., 2004; Song et al., 2015) lobes, as well as in the right parietal lobe (Maruff et al., 2004). They are also associated with WM volume reduction in the medial temporal lobe (Makris et al., 2010). Few studies, nonetheless, have explored the neural correlates of specific types of delusions, mostly delusions of control, also called Schneiderian or passivity delusions, which might share certain neural underpinnings with AVH because of their shared self-agency defect component (Poletti et al., 2017).

\subsection{Negative symptoms}

With regard to negative symptoms, they have been associated with GM volume decrement in medial frontal and orbitofrontal areas in patients with schizophrenia (see Kaladjian et al., 2015 for a review). An association of negative symptoms with WM abnormalities has also been reported. This includes WM abnormalities in the superior longitudinal fasciculus in patients with first-episode psychosis (Sun et al., 2015) and with schizophrenia (Chuang et al., 2014). Further, avolition and anhedonia in patients with schizophrenia have been related to WM reduction in the corpus callosum (Nakamura et al., 2012), left posterior medial orbitofrontal cortex, and left anterior cingulate cortex (Ohtani et al., 2014a). Another study found reduced WM volume in the cingulate region to be associated with alogia (Makris et al., 2010).

\subsection{Affective symptoms}

There is abundant evidence suggesting that affective conditions have significant associations with the severity, distress and content of psychotic experiences. Anxiety 
and depression, as two specific affective conditions, may be prodromes for psychosis (Hartley et al., 2013). Although the effects of affective symptoms on GM and WM volumes have been studied in patients with depression (Ballmaier et al., 2004; Zhang et al., 2016) and anxiety disorders (Jackowski et al., 2012), only one study, as far as we know, has examined the effect of depression on brain volume in schizophrenia. Results showed that depression severity was associated with lower GM orbitofrontal volume in female patients (Gur et al., 2000).

Considering the high prevalence of depression and anxiety in patients with schizophrenia, it is possible that these symptoms have a significant role in the GM and WM volume reductions observed in this population. More specifically, depression is often observed in patients with auditory-verbal hallucinations and delusions and it is therefore important to tease apart the potential effects of depressive symptoms from those of positive symptoms. Further, given the overlap between depressive and negative symptoms, the extent to which the observed effects of negative symptoms on GM and WM abnormalities might have been influenced by the shared depressive component needs to be assessed.

\subsection{Aims of the study}

In this study we identified brain areas in which GM or WM volume reduction was observed in patients with schizophrenia, and we sought to determine the clinical symptoms that were involved in these volume reductions. The targeted symptoms were $\mathrm{AVH}$, Schneiderian and non-Schneiderian delusions, negative symptoms, and affective symptoms.

\section{Methods}

The involved institutions approved the study protocol in accordance with Declaration of Helsinki (World Medical Association, 2013) guidelines. All procedures 
were approved by the Parc Sanitari Sant Joan de Déu ethics committee, and all participants provided written informed consent. All the rating scales were administrated by means of interview by an experienced clinical psychologist.

\subsection{Participants}

Twenty-seven healthy participants were recruited from the general population by means of announcements, although one participant was eventually excluded due to the poor quality of the MRI images. Inclusion criteria were age between 18 and 60 years and fluency in Spanish. Exclusion criteria were neurological or mental illness, intellectual disability, head injury, alcohol or drug abuse in the previous six months, and current severe physical disease, as well as the standard exclusion criteria for MRI procedures: claustrophobia and metallic implants (fitted pacemaker and cochlear implants).

Twenty-four patients with schizophrenia (American Psychological Association (APA), 2002) were recruited from the Parc Sanitari Sant Joan de Déu network of mental health services in Barcelona, Spain. All patients were receiving antipsychotic treatment. Inclusion criteria were the same as those for healthy participants with the additional criterion of being able to provide informed consent. Exclusion criteria were also the same except that the mental disease exclusion criterion only applied to organic mental disorders and dementia.

\subsection{Clinical rating scales}

Positive and negative symptoms were assessed in patients using the Spanish version of the Scale for the Assessment of Positive Symptoms (SAPS) (Andreasen, 2000) and the Scale for the Assessment of Negative Symptoms (SANS)(Andreasen, 1989; Peralta and Cuesta, 1999). With respect to positive symptoms, an AVHs score was computed by summing the scores obtained on the $2^{\text {nd }}$ and $3^{\text {rd }}$ items of the SAPS hallucination scale ('voices commenting' and 'voices conversing'). A Schneiderian 
delusions (Schneider, 1959) (delusions of mind reading and of being controlled, thought broadcasting, thought insertion, thought withdrawal) score, consisting of the sum of the $15^{\text {th }}-19^{\text {th }}$ (5 items), and a 'non-Schneiderian delusions' score consisting of the sum of the remaining delusion items (delusions of persecution, jealousy, guilt or sin, religious, reference, grandiose, and somatic) were also computed. Negative symptom scores were tallied for flat affect, alogia, avolition, and anhedonia. Depression was assessed using the Calgary Depression Scale, specifically designed to assess depression in schizophrenia and differentiate it from negative symptoms (Addington et al., 1992; Sarro et al., 2004). Anxiety was measured using the Hamilton Anxiety Rating Scale (Hamilton, 1959; Lobo et al., 2002). Clinical variables that did not follow normal distribution were normalized by square-root transformation. Chlorpromazine equivalence was calculated for antipsychotic drug doses according to Gardner (Gardner et al., 2010).

\subsection{MRI Data Acquisition}

MRI data were acquired using a General Electric 1.5 Tesla SignaHDe scanner at Parc Sanitari Sant Joan de Déu. High-resolution T1-weighted FSPGR structural images with the axial plane parallel to the AC-PC axis were acquired using the following parameters: 2 mm slice thickness, $\mathrm{TR}=12.24 \mathrm{~ms}, \mathrm{TE}=3.84 \mathrm{~ms}, \mathrm{FOV}=24 \mathrm{~cm}$, acquisition matrix $=512 \times$ 512, flip angle $=20^{\circ}$, voxel size $=0.47 \times 0.47 \times 2.0 \mathrm{~mm}^{3}$ (individual voxel volume: 0.442 $\left.\mathrm{mm}^{3}\right)$.

\subsubsection{Preprocessing of imaging data}

Preprocessing followed the default procedures used in the VBM8 toolbox http://dbm.neuro.uni-jena.de/vbm8/VBM8-Manual.pdf, running within the Statistical Parametric Mapping software package version 8

(SPM8; http://www.fil.ion.ucl.ac.uk/spm), using MATLAB (R2010b, The MathWorks, Inc., Natick, Massachusetts). We normalized T1 images to a template space provided by the 
Montreal Neurological Institute (MNI) stereotactic space, which includes highdimensional DARTEL (Diffeomorphic Anatomic Registration Through Exponentiated Lie) algebra normalization (Ashburner, 2007). Subsequently, we segmented into GM, WM, and cerebrospinal fluid (CSF) and performed the non-linear modulation step (the modulation step multiplies the spatially normalized images by their relative volume before and after spatial normalization). The final GM and WM were extracted by segmenting the individual raw T1 images using standard GM, WM and CSF probability maps and then smoothed using an 8-mm full-width at half maximum Gaussian kernel.

\subsection{Statistical analysis}

\subsubsection{Descriptive analysis}

Means with standard deviations are reported for continuous variables. Counts and percentages are reported for categorical variables. Differences by groups in continuous variables were explored with t-test or ANCOVA, whenever appropriate. Categorical analyses were done with the $x^{2}$ test. Pearson correlation was used to explore the associations between the depression and negative scores (SANS total). All tests were performed with SPSS version 21 (IBM Corp. Released, 2012).

\subsubsection{Voxel-based morphometry analysis}

We performed a data-quality check to ensure sample homogeneity prior to the analyses with the VBM8 toolbox. This procedure was executed using the 'check sample homogeneity' function. Following this, the raw volumes for GM, WM and CSF, and total intracranial volumes (the sum of GM, WM and CSF) of the segmented raw images were calculated. To identify regions with volume reduction in patients, we conducted a between-group comparison by means of a t-test, adding age and sex as covariates due to their potential confounding effect on this association (Kaufmann et al., 2001; Núñez et al., 2018). To further reduce the potential effects of tissue overlap occurring at tissue 
border regions, we used, separately, the implicit GM and WM masks included in SPM8. We calculated GM and WM volume differences between groups separately using a cluster-defining voxel-level threshold (CDT) defined by $\mathrm{p}<0.001$ together with a familywise-error (FWE) corrected cluster extent threshold $\mathrm{p}<0.05$. A more restrictive CDT $\mathrm{p}<0.0001$ and cluster level FWE-corrected $\mathrm{p}<0.05$ were applied if the clusters generated by the more permissive CDT spanned several brain regions. For both cases, individual patient tissue density scores were extracted from the volumes of interest (VOI) defined by the statistically significant clusters. Then, to better identify the individual brain structures within clusters encompassing several brain regions, a more restrictive voxelwise threshold $p<0.0001$, followed by cluster level FWE-corrected with $p<0.05$ were applied.

\subsubsection{Potential confounders}

To identify potentially confounding factors in the associations with clinical symptoms, we conducted regression analyses on the GM and WM VOIs identified in the patient group. The predictors were the socio-demographic measures (age, sex, education and verbal IQ), antipsychotic dosage, and illness duration. Regression analyses were also conducted in the healthy sample using solely the socio-demographic measures as predictors to determine whether potential associations were similarly observed in this group.

\subsubsection{Associations with clinical symptoms in the patient group}

The associations between GM and WM permissive CDT $(p<0.001)$ and clinical symptoms were investigated by means of regression analyses. We first conducted a regression analysis using the four negative symptoms (flat affect, alogia, avolition and anhedonia) along with the confounding factors identified in the previous step. The backward method was used to eliminate step by step the factors which did not 
contribute to the model. Subsequently, another regression analysis was performed with the affective (depression and anxiety) and positive (AVH, Schneiderian and nonSchneiderian delusions) symptom scores entered as predictors along with the contributors identified in the previous step. Again, the backward method was used to eliminate non-associated variables. The final model resulting from this latter regression analysis was then used on each of the brain structures identified with a more restrictive cluster-defining threshold $p<0.0001$, so as to investigate the associations between the clinical symptoms of interest and more specific brain areas.

\section{Results}

Baseline demographics and clinical characteristics of patients and healthy controls are summarized in Table 1. Depression score was highly significantly correlated with SANS score $(r=0.69, p<0.0001)$, which suggests an overlap between these two scales.

Insert Table 1

\subsection{Total brain volume and identification of regions with significant deficit in patients}

There were significant GM volume differences, but not for WM, CFS and total intracranial volume between groups (see table A1 in supplementary materials). We observed decreased GM and WM volume and increased cerebrospinal fluid volume in schizophrenia patients compared with healthy controls.

A comparison of patients with schizophrenia and healthy subjects, made using a permissive CDT $p<0.001$ followed by an FWE-corrected $p<0.05$, revealed reduced GM and WM volumes in the prefrontal cortex in patients. The following brain structures (identified with a more restrictive cluster-defining threshold $p<0.0001$ ) showed a GM reduction in patients: bilateral superior frontal gyrus, right medial superior frontal 
gyrus and middle frontal gyrus, right medial orbitofrontal cortex and bilateral rectus gyrus while reductions in WM (identified with a more restrictive CDT $p<0.0001$ ) were in the tract adjacent to the right superior frontal gyrus and in the left body of the corpus callosum. In the case of GM, mean densities were obtained over a 274 voxel-sized cluster remaining after FWE correction of a restrictive CDT $p<0.0001$. In the case of WM, mean densities were obtained over a 553-voxel cluster remaining after FWE correction at the cluster level (see Table 2, Figure 1).

Insert Table 2

\subsection{Associations between prefrontal GM and WM volume reduction and socio-demographic} measures, antipsychotic dosage, and illness duration

\subsubsection{GM}

In the patient group, the model with the 6 predictors $\left(R^{2}=0.69, F(6,23)=6.20\right.$, $p<0.001)$ revealed a significant negative contribution of age $(\beta=-0.627, p=0.015)$ and a trend level positive contribution of verbal IQ $(\beta=0.369, p=0.08)$ to the GM prefrontal VOI resulting from permissive CDT $p<0.001$. Education level, illness duration and antipsychotic dosage were not significantly related to GM volume $(p>0.10)$. Sex was a significant predictor $(\beta=0.489, p=0.015)$ and examination of the data showed that women had larger prefrontal volume than men (estimated marginal means: 0.46 vs. $0.42)$.

In the control group, the model with 4 predictors $\left(R^{2}=0.56, F(4,25)=6.73\right.$, $p<0.001$ ) similarly revealed a negative association with age, although only at a trend level of significance $(\beta=-0.350, p=0.063)$. Sex was again a significant predictor $(\beta=0.366$, 
$p=0.028$ ) and, as was observed in patients, women had larger frontal volumes than men (estimated marginal means: 0.50 vs. 0.47 ).

To determine whether women's volume superiority was equivalent in both groups, we compared the prefrontal GM volume resulting from permissive CDT of patients and controls using a univariate general model with age, education level, and verbal IQ as covariates. A strong effect of sex was revealed $(F(1.43)=13.9, p<0.001)$ without any interaction with group $(F<1, p=0.60)$.

\subsubsection{WM}

No association between the WM prefrontal VOI resulting from permissive CDT and any of the four socio-demographic factors was observed in either group, and no association with antipsychotic dosage or illness duration was observed in the patient group.

\subsection{Associations of frontal GM and WM volume with clinical symptoms in patients}

\subsection{1. $G M$}

Regression analysis on GM VOI, including the four negative symptom scores along with age, sex and verbal IQ, and using the backward method, resulted in a final model with a significant contribution of anhedonia $(\beta=-0.30, p<0.025)$ along with age, sex, and verbal IQ. These four factors were entered into a subsequent regression analysis, along with the positive and affective symptoms. The contribution of anhedonia was no longer observed. The backward method resulted in a significant 5-factor model with significant contributions of age, sex, AVHs and depression, and a trend level contribution of non-Schneiderian delusions (see Table 3). It should be noted that the 'Schneiderian delusion' score made a near-zero contribution to the model and was the first factor to be eliminated by the backward method. 
Regression analyses using this 5-factor model were then conducted on each of the GM VOIs resulting from a restrictive CDT. Results are presented in Table 3. AVHs tended to be negatively associated with the right medial superior frontal and right orbitofrontal gyrus, while the association with the left superior frontal gyrus was near zero. The depression score was inversely associated with the right medial superior frontal and right orbitofrontal gyrus, and, at a trend level, with the right middle frontal and superior frontal gyrus (Figure 2).

Insert Table 3

\subsection{2. $W M$}

The first regression analysis, including only the four negative symptoms, revealed significant contributions of anhedonia and alogia to the WM prefrontal VOI (resulting from the permissive CDT) in the final model. Anhedonia and alogia were then entered into a second regression analysis along with positive (AVH, Schneiderian delusions, non-Schneiderian delusions) and affective (depression, anxiety) symptom scores. The AVHs score did not make any contribution to the WM VOI ( $\beta$ near zero) and it was the first factor to be eliminated by the backward method. The last model revealed significant contribution of anhedonia, alogia, and non-Schneiderian delusions. When the Schneiderian delusion score was entered into this last model instead of the nonSchneiderian score, it also made a trend significant contribution to WM, suggesting that both type of delusions were associated with WM reduction. We therefore used a final model with anhedonia, alogia, and global delusion score (see Table 4).

Regression analyses using this 3-factor model were then conducted on each of the specific clusters identified. The global delusion score made a significant negative contribution to the right superior frontal WM cluster, again with contribution of both 
Schneiderian $(\beta=-0.338, p<0.07)$ and non-Schneiderian $(\beta=-0.362, p<0.05)$ delusions.

Insert Table 4

\section{Discussion}

Various studies of schizophrenia (Fornito et al., 2009; Y. Zhang et al., 2016) and individuals at high risk of psychosis (Gogtay et al., 2011) have revealed decrements in the frontal cortex relative to healthy controls. In line with these, we observed reduced GM volume in various areas of the prefrontal cortex in our patient sample. Further, WM volume reduction was observed in the tract adjacent to the right superior frontal gyrus and the body of the corpus callosum, in agreement with other studies (Collinson et al., 2014; Knöchel et al., 2012; Nakamura et al., 2012; Ohtani et al., 2014a). These volume decrements in both GM and WM were found to be differentially associated with specific positive, affective, and negative symptoms.

\subsection{AVHs}

AVHs were significantly associated with decreased GM in the prefrontal cortex. In particular, they were associated, although only at trend level, with decreased GM in a brain area that encompasses the right medial superior frontal and medial orbitofrontal cortex. Although research has mostly documented a relationship between severity of AVHs and GM reduction in the left superior temporal gyrus in schizophrenia patients (Modinos et al., 2013; O’Daly et al., 2007; Rajarethinam et al., 2000; Sun et al., 2009), this 
type of hallucination has also been related to GM reduction in the right prefrontal gyrus in previous research (Gaser et al., 2004; Modinos et al., 2009; Zmigrod et al., 2016), in agreement with our data. This association with prefrontal cortex volume may underlie the link between AVHs and certain executive functions, namely working memory (Brébion et al., 2015; Daalman et al., 2011; Gisselgård et al., 2014), set-shifting (Siddi et al., 2017; Waters et al., 2012), and semantic fluency (DeFreitas et al., 2009) observed in several studies.

\subsection{Delusions}

Delusions too have been found to be associated with reduced frontal GM volume in previous research (Maruff et al., 2004; Song et al., 2015). However, this association may partly result from overlap between AVH and a certain type of delusions, namely Schneiderian delusions. Indeed, both AVH and Schneiderian delusions are related to a lack of the sense of self-agency (Poletti et al., 2017), which might be subserved by the prefrontal cortex. In our data, non-Schneiderian delusions tended, on the contrary, to be associated with increased prefrontal GM volume. Their neural underlying mechanism, thus, seems to be entirely different from that of AVHs. Further, both Schneiderian and, at a trend level, non-Schneiderian delusions were associated with decreased prefrontal WM, particularly in the right superior frontal gyrus. WM alterations in various neural regions including the frontal lobe may lead to deficits in social cognition (Wang and Olson, 2018), which might be a cognitive underpinning of various types of delusions (Park, 2018). Delusions have also been found to be associated with WM reduction in the medial temporal lobe in patients with schizophrenia (Makris et al., 2010). These findings may reflect involvement of both GM and WM in various types of delusions.

\subsection{Depression}


Although depressive symptoms are highly prevalent in patients with schizophrenia, they are not commonly taken into account in neural studies in this population. Our results suggest nonetheless that depression has a significant impact on GM volume reduction, which might have been overlooked in previous research and confounded by the effects of negative symptoms. Indeed, in our data, the contribution of anhedonia, which emerged in the first step of the regression analysis, was no longer significant when depressive symptoms were entered into the model. Similarly, the effects of negative symptoms on GM reported in numerous studies might partly stem from overlap between negative and depressive symptoms.

Our findings indicate that depression had a significant impact on GM reduction in the prefrontal cortex, more specifically in the right medial superior frontal, medial orbitofrontal, and superior frontal and middle frontal gyrus. This observation is in agreement with that of Gur et al. (2000) who reported that lower GM orbitofrontal volume was associated with greater depression severity in schizophrenia, although this was only observed in women in their study.

The orbitofrontal cortex is essential for the interaction between emotional valence and self-monitoring, as well as social behavior (Beer et al., 2006), and the detrimental effect of depression on this brain area might participate in the selfmonitoring disturbance that leads to AVHs (Waters et al., 2012). In addition, this impact of depression on GM prefrontal volume probably explains the adverse effects of depression on cognitive performance in this population. In particular, it was observed in several schizophrenia samples that depression severity affected memory efficiency (Brébion et al., 2013; Sanfilipo et al., 2002) and specifically impacted the deep/effortful processes of verbal encoding (Brébion et al., 2001, 2000; Lysaker et al., 2000; Möser et al., 2006), allegedly subserved by the prefrontal cortex. Thus, this neuroimaging study confirms, at the brain structural level, the previous observation that depression in these 
patients is a crucial impairment factor in the memory tasks that rely upon executive processes.

Thus, our findings evince a critical involvement of depression in the brain dysfunction of patients with schizophrenia, with potential consequences for the clinical and cognitive manifestations of the disease. It should be noted that the average level of depression in our patient sample was low. More striking results might have emerged in a sample with more significant levels of depression.

\subsection{Negative symptoms}

When depression was taken into account, none of the negative symptoms we investigated was found to have any impact on GM volume reduction. In contrast, negative symptoms appeared to be significantly related to WM abnormalities. Anhedonia and alogia presented significant and opposite associations with WM volume in the prefrontal cortex, more specifically in the tract adjacent to the right superior frontal gyrus. The right superior frontal gyrus is involved in motivational processes (Spielberg et al., 2008). Previous studies have reported WM abnormalities in the frontal lobe to be associated with the negative symptoms of schizophrenia (Chuang et al., 2014; Ohtani et al., 2014a). In our data, anhedonia was further associated with WM volume reduction in the left corpus callosum. In line with this, WM alterations in the corpus callosum have been found to be associated with alogia (Makris et al., 2010), avolition (Nakamura et al., 2012) and anhedonia (Lee et al., 2014) in patients with schizophrenia.

Thus, negative and depressive symptoms appear to be subserved by distinct structural abnormalities. This neural distinction may reflect the phenomenological distinction between them: low mood, suicidal ideation, and pessimism have more specificity for depression, whereas alogia and blunted affect may have more specificity as negative symptoms (Krynicki et al., 2018). 


\subsection{Limitations}

The main limitation of this study is the small size of the patient sample. Further, we did not control certain factors that might have had some influence on the results, such as handedness, and a possible family-related risk of mental illness in the healthy participants.

\section{Conclusions}

Our findings reveal the important role of depression in GM prefrontal volume decrement in schizophrenia. Depression appears to impact the same brain areas as AVHs, and it may therefore have a critical role in the formation of voices, which are one of the most common and distressing symptoms of the disease. Further, depression emerged as a more significant factor of GM prefrontal volume reduction than negative symptoms. The potential effects of depressive symptoms should be more routinely taken into account in this type of investigation in order to better understand the pathological mechanisms of schizophrenia. Depression in schizophrenia has detrimental consequences, such as high relapse rate, suicidal ideation, increased mortality, reduced social adjustment and poor quality of life (Krynicki et al., 2018). Longitudinal investigations reported that depression can predate first-episode psychosis, or acute relapses, which may indicate their causal influence or role in triggering distressing experiences (Hartley et al., 2013). Our findings indicate that the impact of depression in patients with schizophrenia can also been evinced at the cerebral level.

\section{Acknowledgments}

The authors wish to thank all the psychiatrists and participants involved in this study. Authors thank Stephen Kelly for his help with the English revision.

\section{Contributors}


SS managed the literature search and undertook the statistical analyses. She wrote the first draft and the final manuscript. GB and CS-O designed the study and wrote the protocol. GB and CS-O were the guarantors of the work: they had full responsibility for the work and the conduct of the study, had access to the data, and controlled the decision to publish. All authors contributed to the organization of the study, and to data collection and analysis, and they all approved the manuscript for submission.

\section{Declarations of interest:}

None

\section{Role of the funding source}

This work was supported by a Miguel Servet contract (CP09/00292) and a grant PI10/02479 from the Instituto de Salud Carlos III - Subdirección General de Evaluación y Fomento de la Investigación Sanitaria - co-funded by Fondo Europeo de Desarrollo Regional (FEDER), both to GB; a grant PRRMAB-A2011-19251 from the 'Master and Back program', Sardinia Region to SS; and a contract PTA2011-4983-I from the Ministerio de Ciencia e Innovación, Spain to CS-O.

\section{References}

Addington, D., Addington, J., Maticka-Tyndale, E., Joyce, J., 1992. Reliability and validity of a depression rating scale for schizophrenics. Schizophr. Res. 6, 201-208. doi:10.1016/0920-9964(92)90003-N

American Psychological Association (APA), 2002. DSM IV-TR, DSM-IV-TR manual diagnóstico y estadístico de los trastornos mentales. MASSON, S.A, Barcelona.

Andreasen, N.C., 1989. Scale for the assessment of negative symptoms (SANS). Br. J. Psychiatry 155, 53-58.

Andreasen, N.C., 2000. Scale for the assessment of positive symptoms. Medicine (Baltimore). 1984, 1-21.

Ashburner, J., 2007. A fast diffeomorphic image registration algorithm. Neuroimage 38, 95-113.

Ballmaier, M., Toga A.W., Arthur W, Blanton, R.E., Sowell, E.R., Lavretsky, H., et al., 2004. Anterior cingulate, gyrus rectus, and orbitofrontal abnormalities in elderly depressed patients: an MRI-based parcellation of the prefrontal cortex. Am. J. 
Psychiatry 161, 99-108.

Beer, J.S., John, O.P., Scabini, D., Knight, R.T., 2006. Orbitofrontal cortex and social behavior: integrating self-monitoring and emotion-cognition interactions. J. Cogn. Neurosci. 18, 871-879. doi:10.1162/jocn.2006.18.6.871

Brébion, G., Amador, X., Smith, M., Malaspina, D., Sharif, Z., Gorman, J.M., 2000. Depression, psychomotor retardation, negative symptoms, and memory in schizophrenia. Neuropsychiatry. Neuropsychol. Behav. Neurol. 13, 177.

Brébion, G., Gorman, J.M., Malaspina, D., Sharif, Z., Amador, X., 2001. Clinical and Cognitive Factors Associated With Verbal Memory Task Performance in Patients With Schizophrenia. Am. J. Psychiatry 158, 758-764. doi:10.1176/appi.ajp.158.5.758

Brébion, G., Villalta-Gil, V., Autonell, J., Cervilla, J., Dolz, M., Foix, A., et al., 2013. Cognitive correlates of verbal memory and verbal fluency in schizophrenia, and differential effects of various clinical symptoms between male and female patients. Schizophr. Res. 147, 81-85. doi:10.1016/j.schres.2013.03.014

Brébion, Stephan-Otto, C., Usall, J., Huerta-Ramos, E., Perez Del Olmo, M., CuevasEsteban, J., et al., 2015. Association of auditory-verbal and visual hallucinations with impaired and improved recognition of colored pictures. Neuropsychology 29, 667-674. doi:10.1037/neu0000169

Chuang, J.-Y., Murray, G.K., Metastasio, A., Segarra, N., Tait, R., Spencer, J., et al., 2014. Brain structural signatures of negative symptoms in depression and schizophrenia. Front. psychiatry 5, 116. doi:10.3389/fpsyt.2014.00116

Collinson, S.L., Gan, S.C., Woon, P.S., Kuswanto, C., Sum, M.Y., Yang, G.L., et al., 2014. Corpus callosum morphology in first-episode and chronic schizophrenia: Combined magnetic resonance and diffusion tensor imaging study of Chinese Singaporean patients. Br. J. Psychiatry 204, 55-60. doi:10.1192/bjp.bp.113.127886

Crespo-Facorro, B., Nopoulos, P.C., Chemerinski, E., Kim, J.J., Andreasen, N.C., Magnotta, V., 2004. Temporal pole morphology and psychopathology in males with schizophrenia. Psychiatry Res. - Neuroimaging 132, 107-115. doi:10.1016/j.pscychresns.2004.09.002

Daalman, K., van Zandvoort, M., Bootsman, F., Boks, M., Kahn, R., Sommer, I., 2011. Auditory verbal hallucinations and cognitive functioning in healthy individuals. Schizophr. Res. 132, 203-207. doi:10.1016/j.schres.2011.07.013

DeFreitas, C.M., Dunaway, L.A., Torres, I.J., 2009. Preferential semantic fluency impairment is related to hallucinations, but not formal thought disorder. Schizophr. Res. 107, 307-312. doi:10.1016/j.schres.2008.08.020

Fornito, A., Yücel, M., Patti, J., Wood, S.J., Pantelis, C., 2009. Mapping grey matter reductions in schizophrenia: An anatomical likelihood estimation analysis of voxelbased morphometry studies. Schizophr. Res. 108, 104-113. doi:10.1016/j.schres.2008.12.011

García-Martí, G., Aguilar, E.J., Lull, J.J., Martí-Bonmatí, L., Escartí, M.J., Manjón, J. V, et al., 2008. Schizophrenia with auditory hallucinations: a voxel-based morphometry study. Prog. Neuropsychopharmacol. Biol. Psychiatry 32, 72-80. doi:10.1016/j.pnpbp.2007.07.014

Gardner, D.M., Murphy, A.L., O’Donnell, H., Centorrino, F., Baldessarini, R.J., 2010. 
International consensus study of antipsychotic dosing. Am. J. Psychiatry 167, 686693. doi:10.1176/appi.ajp.2009.09060802

Gaser, C., Nenadic, I., Volz, H.-P., Büchel, C., Sauer, H., 2004. Neuroanatomy of "hearing voices": a frontotemporal brain structural abnormality associated with auditory hallucinations in schizophrenia. Cereb. Cortex 14, 91-96.

Gisselgård, J., Anda, L.G., Brønnick, K., Langeveld, J., Ten Velden Hegelstad, W., Joa, I., et al., 2014. Verbal working memory deficits predict levels of auditory hallucination in first-episode psychosis. Schizophr. Res. 153, 38-41.

doi:10.1016/j.schres.2013.12.018

Gogtay, N., Vyas, N.S., Testa, R., Wood, S.J., Pantelis, C., 2011. Age of onset of schizophrenia: perspectives from structural neuroimaging studies. Schizophr. Bull. 37, 504-513. doi:10.1093/schbul/sbr030

Gur, R.E.C., Cowell, P.E., Latshaw, A., Turetsky, B.I., Grossman, R.I., Arnold, S.E., et al., 2000. Reduced dorsal and orbital prefrontal gray matter volumes in schizophrenia. Arch Gen Psychiatry 57, 761-768. doi:10.1001/archpsyc.57.8.761

Haijma, S. V, Van Haren, N., Cahn, W., Koolschijn, P.C., Hulshoff Pol, H.E., Kahn, R.S., 2013. Brain volumes in schizophrenia: a meta-analysis in over 18000 subjects. Schizophr. Bull. 39, 1129-38. doi:10.1093/schbul/sbs118

Hamilton, M., 1959. The assessment of anxiety states by rating. Br. J. Med. Psychol. 32, 50-5.

Hartley, S., Barrowclough, C., Haddock, G., 2013. Anxiety and depression in psychosis: A systematic review of associations with positive psychotic symptoms. Acta Psychiatr. Scand. 128, 327-346. doi:10.1111/acps.12080

IBM Corp. Released, 2012. IBM SPSS Statistics for Mac, Version 21.0. 2012.

Jackowski, A.P., Araújo Filho, G.M. de, Almeida, A.G. De, Araújo, C.M. De, Reis, M., Nery, F., et al., 2012. The involvement of the orbitofrontal cortex in psychiatric disorders: an update of neuroimaging findings. Rev. Bras. Psiquiatr. 34, 207-212. doi:10.1590/S1516-44462012000200014

Kaladjian, A., Belzeaux, R., Adida, M., Azorin, J.M., 2015. Symptômes négatifs et imagerie cérébrale. Negative symptoms and cerebral imaging. Encephale 41, 6S22-6S26. doi:10.1016/S0013-7006(16)30006-9

Kaufmann, C., Wilke, M., Pütz, B., Auer, D.P., 2001. Gender differences in brain morphology: voxel based morphometry in 102 healthy young women and men. Proc. Intl. Soc. Mag. Reson. Med 9, 1373.

Knöchel, C., O’Dwyer, L., Alves, G., Reinke, B., Magerkurth, J., Rotarska-Jagiela, A., et al., 2012. Association between white matter fiber integrity and subclinical psychotic symptoms in schizophrenia patients and unaffected relatives. Schizophr. Res. 140, 129-35. doi:10.1016/j.schres.2012.06.001

Krynicki, C.R., Upthegrove, R., Deakin, J.F.W., Barnes, T.R.E., 2018. The relationship between negative symptoms and depression in schizophrenia: a systematic review. Acta Psychiatr. Scand. 1-11. doi:10.1111/acps.12873

Kubera, K.M., Sambataro, F., Vasic, N., Wolf, N.D., Frasch, K., Hirjak, D., et al., 2014. Source-based morphometry of gray matter volume in patients with schizophrenia who have persistent auditory verbal hallucinations. Prog. NeuroPsychopharmacology Biol. Psychiatry 50, 102-109. 
doi:10.1016/j.pnpbp.2013.11.015

Lee, J.S., Han, K., Lee, S.K., Seok, J.H., Kim, J.J., 2014. Altered structural connectivity and trait anhedonia in patients with schizophrenia. Neurosci. Lett. 579, 7-11. doi:10.1016/j.neulet.2014.07.001

Lobo, A., Chamorro, L., Luque, A., Dal-ré, R., Badia, X., Baró, E., 2002. Validación de las versiones en español de la Montgomery-Asberg Depression Rating Scale y la Hamilton Anxiety Rating Scale para la evaluación de la depresión y de la ansiedad. Med. Clin. (Barc). 118, 493-499. doi:10.1016/S0025-7753(02)72429-9

Lysaker, P.H., Bell, M.D., Greig, T.C., Bryson, G.J., 2000. Emotional discomfort and impairments in verbal memory in schizophrenia. Psychiatry Res. 97, 51-59. doi:10.1016/S0165-1781(00)00221-3

Makris, N., Seidman, L.J., Ahern, T., Kennedy, D.N., Caviness, V.S., Tsuang, M.T., Goldstein, J.M., 2010. White matter volume abnormalities and associations with symptomatology in schizophrenia. Psychiatry Res. - Neuroimaging 183, 21-29. doi:10.1016/j.pscychresns.2010.04.016

Maruff, P., Wood, S.J., Velakoulis, D., Smith, D.J., Soulsby, B., Suckling, J., et al., 2004. Reduced volume of parietal and frontal association areas in patients with schizophrenia characterized by passivity delusions. Psychol. Med. 35, 783-789. doi:10.1017/S0033291704003113

Modinos, G., Costafreda, S.G., Van Tol, M.J., McGuire, P.K., Aleman, A., Allen, P., 2013. Neuroanatomy of auditory verbal hallucinations in schizophrenia: A quantitative meta-analysis of voxel-based morphometry studies. Cortex 49, 1046-1055. doi:10.1016/j.cortex.2012.01.009

Möser, C., Krieg, J.C., Zihl, J., Lautenbacher, S., 2006. Attention and Memory Deficits in Schizophrenia: The Role of Symptoms of Depression. Cogn. Behav. Neurol. 19, 150156. doi:wnn.0000213910.41901.1a

Nakamura, K., Kawasaki, Y., Takahashi, T., Furuichi, A., Noguchi, K., Seto, H., Al., E., 2012. Reduced white matter fractional anisotropy and clinical symptoms in schizophrenia: A voxel-based diffusion tensor imaging study. Psychiatry Res. Neuroimaging 202, 233-238. doi:10.1016/j.pscychresns.2011.09.006

Núñez, C., Theofanopoulou, C., Senior, C., Cambra, M.R., Usall, J., Stephan-Otto, C., Al., E., 2018. A large-scale study on the effects of sex on gray matter asymmetry. Brain Struct. Funct. 223, 183-193. doi:10.1007/s00429-017-1481-4

O’Daly, O.G., Frangou, S., Chitnis, X., Shergill, S.S., 2007. Brain structural changes in schizophrenia patients with persistent hallucinations. Psychiatry Res. 156, 15-21. doi:10.1016/j.pscychresns.2007.03.001

Ohtani, T., Bouix, S., Hosokawa, T., Saito, Y., Eckbo, R., Ballinger, T., et al., 2014a. Abnormalities in white matter connections between orbitofrontal cortex and anterior cingulate cortex and their associations with negative symptoms in schizophrenia: A DTI study. Schizophr. Res. 157, 190-197. doi:10.1016/j.schres.2014.05.016

Ohtani, T., Levitt, J.J., Nestor, P.G., Kawashima, T., Asami, T., Shenton, M.E., et al., 2014b. Prefrontal cortex volume deficit in schizophrenia: a new look using 3T MRI with manual parcellation. Schizophr. Res. 152, 184-190.

doi:10.1016/j.schres.2013.10.026 
Palaniyappan, L., Balain, V., Radua, J., Liddle, P.F., 2012. Structural correlates of auditory hallucinations in schizophrenia: A meta-analysis. Schizophr. Res. 137, 169-173. doi:10.1016/j.schres.2012.01.038

Park, S., 2018. A study on the theory of mind deficits and delusions in schizophrenic patients. Issues Ment. Health Nurs. 39, 269-274. doi:10.1080/01612840.2017.1378782

Peralta, V., Cuesta, M.J., 1999. Dimensional structure of psychotic symptoms: an itemlevel analysis of SAPS and SANS symptoms in psychotic disorders. Schizophr. Res. $38,13-26$.

Poletti, M., Gebhardt, E., Raballo, A., 2017. Corollary discharge, self-agency, and the neurodevelopment of the psychotic mind. JAMA psychiatry 74, 1169-1170. doi:10.1001/jamapsychiatry.2017.2824

Rajarethinam, R.P., DeQuardo, J.R., Nalepa, R., Tandon, R., 2000. Superior temporal gyrus in schizophrenia: A volumetric magnetic resonance imaging study. Schizophr. Res. 41, 303-312. doi:10.1016/S0920-9964(99)00083-3

Sanfilipo, M., Lafargue, T., Rusinek, H., Arena, L., Loneragan, C., Lautin, A., et al., 2002. Cognitive performance in schizophrenia: Relationship to regional brain volumes and psychiatric symptoms. Psychiatry Res. - Neuroimaging 116, 1-23. doi:10.1016/S0925-4927(02)00046-X

Sarro, S., Duenas, R.M., Ramirez, N., Arranz, B., Martinez, R., Sanchez, J.M., et al., 2004. Cross-cultural adaptation and validation of the Spanish version of the Calgary Depression Scale for Schizophrenia. Schizophr Res 68, 349-356. doi:10.1016/S0920-9964(02)00490-5\rS0920996402004905 [pii]

Schneider, K., 1959. Clinical psychopathology., 5th editio. ed. Grune \& Stratton, New York.

Seok, J.-H., Park, H.-J., Chun, J.-W., Lee, S.-K., Cho, H.S., Kwon, J.S., et al., 2007. White matter abnormalities associated with auditory hallucinations in schizophrenia: a combined study of voxel-based analyses of diffusion tensor imaging and structural magnetic resonance imaging. Psychiatry Res. 156, 93-104.

doi:10.1016/j.pscychresns.2007.02.002

Siddi, S., Petretto, D.R., Burrai, C., Scanu, R., Baita, A., Trincas, P., et al., 2017. The role of set-shifting in auditory verbal hallucinations. Compr. Psychiatry 74, 162-172. doi:10.1016/j.comppsych.2017.01.011

Song, J., Han, D.H., Kim, S.M., Hong, J.S., Min, K.J., Cheong, J.H., et al., 2015. Differences in gray matter volume corresponding to delusion and hallucination in patients with schizophrenia compared with patients who have bipolar disorder. Neuropsychiatr. Dis. Treat. 11, 1211-1219. doi:10.2147/NDT.S80438

Spielberg, J.M., Stewart, J.L., Levin, R.L., Miller, G.A., Heller, W., 2008. Prefrontal cortex, emotion, and approach/withdrawal motivation. Soc. Personal. Psychol. Compass 2, 135-153. doi:10.1111/j.1751-9004.2007.00064.x

Sun, H., Lui, S., Yao, L., Deng, W., Xiao, Y., Zhang, W., et al., 2015. Two patterns of white matter abnormalities in medication-naive patients with first-episode schizophrenia revealed by diffusion tensor imaging and cluster analysis. JAMA Psychiatry 72, 678-686. doi:10.1001/jamapsychiatry.2015.0505

Sun, J., Maller, J.J., Guo, L., Fitzgerald, P.B., 2009. Superior temporal gyrus volume change 
in schizophrenia: a review on region of interest volumetric studies. Brain Res. Rev. 61, 14-32. doi:10.1016/j.brainresrev.2009.03.004

Van Tol, M.J., Van Der Meer, L., Bruggeman, R., Modinos, G., Knegtering, H., Aleman, A., 2014. Voxel-based gray and white matter morphometry correlates of hallucinations in schizophrenia: The superior temporal gyrus does not stand alone. NeuroImage Clin. 4, 249-257. doi:10.1016/j.nicl.2013.12.008

Wang, Y., Olson, I.R., 2018. The Original social network: white matter and social cognition. Trends Cogn. Sci. 22, 504-516. doi:10.1016/j.tics.2018.03.005

Waters, F., Allen, P., Aleman, A., Fernyhough, C., Woodward, T.S., Badcock, J.C., et al., 2012. Auditory hallucinations in schizophrenia and nonschizophrenia populations: A review and integrated model of cognitive mechanisms. Schizophr. Bull. 38, 683692. doi:10.1093/schbul/sbs045

World Medical Association, 2013. World Medical Association Declaration of Helsinki: ethical principles for medical research involving human subjects. JAMA 310, 2191 4. doi:10.1001/jama.2013.281053

Zhang, H., Li, L., Wu, M., Chen, Z., Hu, X., Chen, Y., et al., 2016. Brain gray matter alterations in first episodes of depression: A meta-analysis of whole-brain studies. Neurosci. Biobehav. Rev. 60, 43-50. doi:10.1016/j.neubiorev.2015.10.011

Zhang, Y., Catts, V.S., Sheedy, D., McCrossin, T., Kril, J.J., Shannon Weickert, C., 2016. Cortical grey matter volume reduction in people with schizophrenia is associated with neuro-inflammation. Transl. Psychiatry 6, e982. doi:10.1038/tp.2016.238 
Table 1. Baseline characteristics of patients and healthy controls

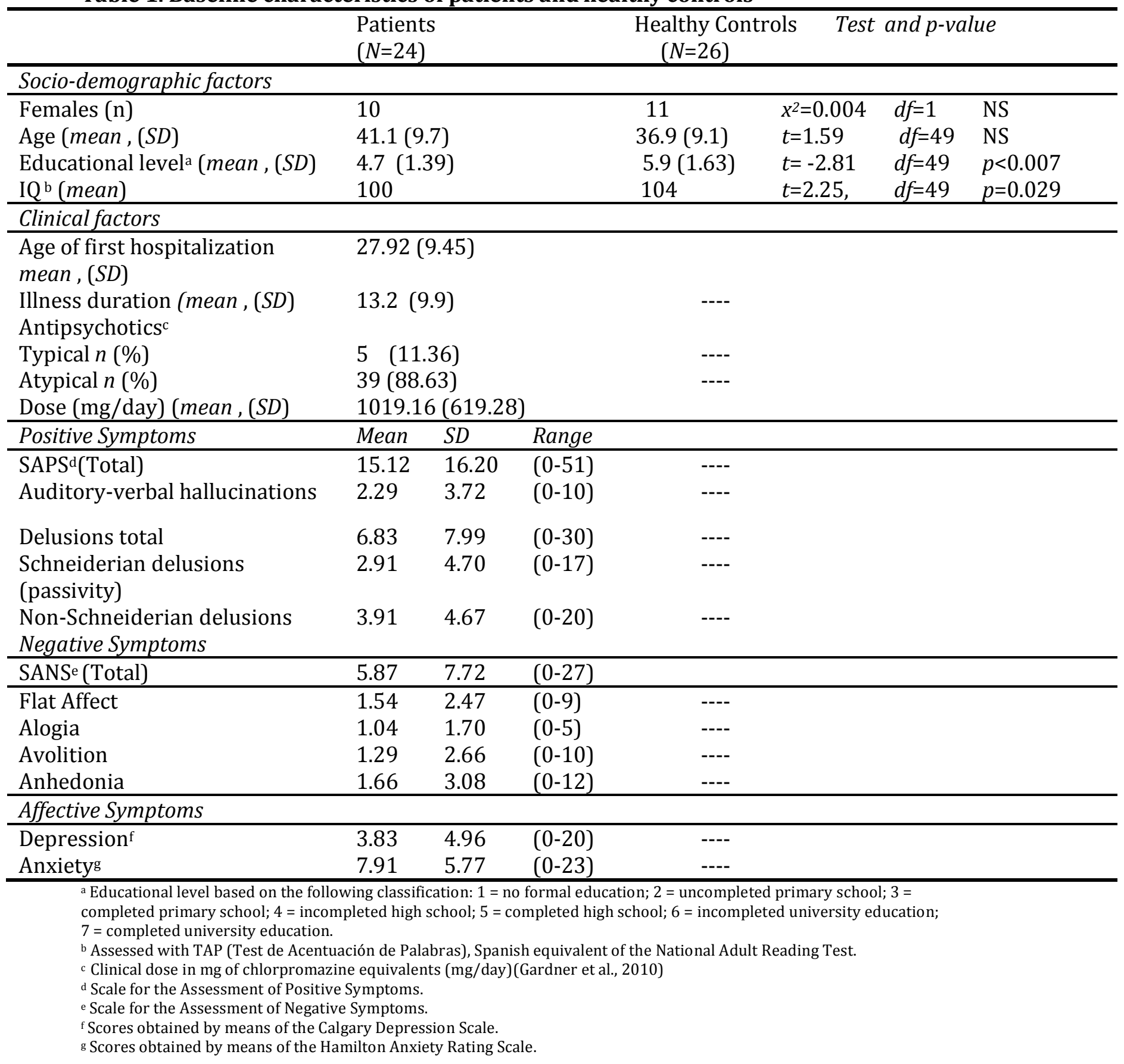


Table 2. Areas with GM and WM density reduction in schizophrenic patients vs. healthy control subjects shown by VBM analysis.

\begin{tabular}{|c|c|c|c|c|c|c|}
\hline \multicolumn{7}{|c|}{ GM } \\
\hline \multicolumn{3}{|c|}{$\begin{array}{l}\text { Coordinates } \\
\end{array}$} & Label & $\mathrm{L} / \mathrm{R}$ & $t$-value & Cluster size \\
\hline$X$ & $Y$ & $z$ & & & & \\
\hline 9 & 70 & 13 & $\begin{array}{l}\text { Medial superior frontal gyrus+ } \\
\text { Medial orbitofrontal gyrus }\end{array}$ & $\mathrm{R}$ & 5.72 & 830 \\
\hline 27 & 60 & 6 & $\begin{array}{l}\text { Middle frontal gyrus + } \\
\text { Superior Frontal gyrus }\end{array}$ & $\mathrm{R}$ & 5.66 & 430 \\
\hline 2 & 45 & -23 & Gyrus rectus & $\mathrm{R} / \mathrm{L}$ & 5.13 & 384 \\
\hline-20 & 11 & 67 & Superior frontal gyrus & $\mathrm{L}$ & 5.01 & 379 \\
\hline \multicolumn{7}{|c|}{ WM } \\
\hline $\bar{X}$ & $\bar{Y}$ & $\bar{Z}$ & & & & \\
\hline 18 & 54 & 15 & Superior frontal gyrus & $\mathrm{R}$ & 5.42 & 459 \\
\hline-14 & -15 & 36 & body of corpus callosum & $\mathrm{L}$ & 5.27 & 1004 \\
\hline
\end{tabular}

Abbreviations: L=left; R=right,

All clusters satisfied FWE cluster-level correction at $\mathrm{p}<0.05$ after a voxel-wise threshold $p<0.001$.

MNI coordinates represent the peak voxel of each cluster where $x, y$, and $z$ indicate the distance measured in millimeters from the anterior commissure in the sagittal, coronal, and axial planes, respectively 
Table 3. Pattern of relationship between GM and clinical symptoms.

\begin{tabular}{|c|c|c|c|c|c|c|c|c|c|}
\hline & AVHs & $\begin{array}{l}\text { Non-Schneid } \\
\text { delusions }\end{array}$ & id. Depression & Age & Sex & $R^{2}$ & $F$ & $d f$ & $p$ \\
\hline $\begin{array}{l}\text { Medial superior frontal g. } \mathrm{R}^{\mathrm{b}} \\
+ \text { +Medial orbitofrontal g. } \mathrm{R}\end{array}$ & $-0.288^{\infty}$ & 0.103 & $-0.317^{*}$ & $-0.697 * * *$ & $0.336^{*}$ & 0.741 & 10.31 & $(5,23)$ & $<0.0001$ \\
\hline $\begin{array}{l}\text { Superior frontal g. and middle } \\
\text { frontal g. R }\end{array}$ & -0.310 & 0.293 & $-0.351^{\infty}$ & -0.122 & $0.395^{\infty}$ & 0.427 & 2.68 & $(5,23)$ & $<0.06$ \\
\hline Rectus gyrus R/L & -0.326 & 0.239 & -0.254 & $-0.521 *$ & 0.036 & 0.391 & 2.31 & $(5,23)$ & $<0.09$ \\
\hline Sup frontal L & -0.053 & 0.149 & -0.144 & $-0.412^{\infty}$ & -0.140 & 0.214 & 0.978 & $(5,23)$ & NS \\
\hline
\end{tabular}

${ }^{* * *} \mathrm{p}<0.0001{ }^{* *} p \leq 0.01,{ }^{*} p \leq 0.05,{ }^{\infty} p<0.09$

Note $=$ A restrictive voxel-wise threshold $\mathrm{p}<0.0001$ and a cluster extent threshold defined by a FWE corrected $\mathrm{p}<0.05$ were applied for each region 
Table 4. Pattern of relationship between WM and clinical symptoms.

\begin{tabular}{llllllll}
\hline & Anhedonia & Alogia & Delusions & $\mathrm{R}^{2}$ & $\mathrm{~F}$ & $\mathrm{df}$ & $\mathrm{p}$ \\
\hline Prefrontal cortex $^{\mathrm{a}}$ & $\mathbf{- 0 . 5 7 9 * * *}^{*}$ & $\mathbf{0 . 4 1 5}^{*}$ & $\mathbf{- 0 . 4 2 6}^{*}$ & 0.462 & 5.72 & $(3,23)$ & 0.005 \\
\hline Super frontal R $^{\mathrm{b}}$ & $\mathbf{- 0 . 5 9 0 * *}^{* *}$ & $\mathbf{0 . 4 1 8}^{*}$ & $\mathbf{- 0 . 4 2 1}^{*}$ & 0.467 & 5.84 & $(3,23)$ & 0.005 \\
Corpus callosum L & $\mathbf{- 0 . 5 9 6 * *}^{*}$ & 0.234 & -0.204 & 0.318 & 3.11 & $(3,23)$ & 0.05 \\
\hline
\end{tabular}

${ }^{*} p<0.05 \quad * * p<0.01 \quad * * * p<0.005$

Note $=$ A restrictive voxel-wise threshold $p<0.0001$ and a cluster extent threshold defined

by a FWE-corrected $p<0.05$ were applied for each region 


\section{Legend to figure}

Figure 1. Multiple axial slices are presented depicting those cerebral areas in which patients with schizophrenia show a significant (A) GM and (B) WM density reduction when compared to healthy controls. A restrictive voxel-level threshold defined by $p<0.0001$ and a cluster extent threshold defined by a $F W E$ corrected $p<0.05$ were used. Each slice is labeled according to the corresponding Z coordinate in the MNI anatomical space.

Figure 2. GM volume abnormalities (red color scale) related to depression and controlled for AVHs, non-Schneiderian delusions, sex and age in the group with schizophrenia. Top Left: GM volume reduction in the rectus gyrus was negatively associated with depression $\left(R^{2}=0.391\right)$. Top right: GM volume reduction in the Medial superior frontal and orbitofrontal gyrus right negatively related to depression $\left(R^{2}=\right.$ 0.741). A restrictive voxel-level threshold defined by $p<0.0001$ and a cluster extent threshold defined by a FWE corrected $p<0.05$ was used. Each slice is labeled according to the corresponding $Z$ coordinate in the MNI anatomical space. 